\title{
Causes of Sleep Complaints
}

Editors

KEITH ROMEO A. AGUILERA

AGNES T. REMULLA

\section{SLEEP MEDICINE CLINICS}

www.sleep.theclinics.com

March 2022 • Volume 17 • Number 1 